\title{
Allergic Reaction Induced Brainstem Stroke in a Patient With Moyamoya Disease : A Case Report
}

\author{
Si Hyun Rho, Hyo Joon Kim \\ Department of Neurosurgery, Presbyterian Medical Center, Jeonju, Korea
}

Received: September 12, 2021

Revised: September 24, 2021

Accepted: September 27, 2021

Corresponding Author:

Hyo Joon Kim, MD, PhD

Department of Neurosurgery,

Presbyterian Medical Center, 365,

Seowon-ro, Wansan-gu, Jeonju

54987, Korea

Tel: 82-63-230-1420

Fax: 82-63-230-1426

Email: hj-kim@hanmail.net

\begin{abstract}
A patient who ate peaches and developed urticaria complained of left sided weakness within minutes. Upon admission, the vital signs and breathing pattern became stable without evidence of anaphylaxis. After magnetic resonance imaging evaluation, the patient was diagnosed with acute ischemic stroke in the right pons, and cerebral angiography revealed moyamoya disease. Reports regarding an allergic reaction without anaphylaxis causing a stroke were extremely limited; however, no study has reported that allergy can cause a stroke in patients with moyamoya to date. Therefore, an ischemic stroke may occur in a patient with moyamoya only with an allergic reaction considered and reported as something that should be considered significant in the daily routine and medication for a patient with moyamoya.
\end{abstract}

Keywords: Hypersensitivity; Ischemic stroke; Moyamoya disease; Anaphylaxis

\section{INTRODUCTION}

Moyamoya disease is characterized by progressive peripheral stenosis of the internal carotid artery (ICA) and its proximal branches and can cause both ischemic and hemorrhagic strokes ${ }^{1)}$. However, its underlying pathophysiology has not yet been fully elucidated, and this poor knowledge reflects the uncertainty and heterogeneity of patient management ${ }^{2}$. To date, no pharmacological treatment has been found to reverse the gradual loss in the ICA. Antiplatelet agents are commonly prescribed for ischemic moyamoya disease, although the coexistence of bleeding risk should be considered $^{3)}$.

Allergy-induced anaphylactic reactions can result in vasodilation, hypotension, bronchospasm, and angioedema, and are characterized by a rapid onset of respiratory and circulatory disturbances ${ }^{4)}$. This can lead to cerebrovascular collapse; however, such cases are rarely reported ${ }^{5)}$. In this case report, an allergic reaction was found to result in an ischemic brainstem stroke in patients with moyamoya disease and poor cerebrovascular conditions, although they are not anaphylactic conditions.

\section{CASE REPORT}

This 69-year-old patient, despite knowing her peach allergy, developed urticaria on her back and left sided weakness after eating peaches, and presented to the emergency room. On admission, the blood pressure and pulse rate were $136 / 86 \mathrm{mmHg}$ and 79 beats per min, respectively. On the first arterial blood gas analysis, the partial pressures of oxygen and carbon dioxide were $78.0 \mathrm{mmHg}$ and $38.0 \mathrm{mmHg}$, respectively. Although the patient had diabetes as an underlying disease, the blood glucose level was $154 \mathrm{mg} / \mathrm{dL}$. The skin had urticaria in the form of red rashes on the back, which were neither pale nor cold. The Glasgow coma scale score was 14

Copyright (C) 2021 The Korean Neurointensive Care Society

This is an Open Access article distributed under the terms of the Creative Commons Attribution Non-Commercial License (http://creativecommons.org/licenses/by-nc/4.0/) which permits unrestricted non-commercial use, distribution, and reproduction in any medium, provided the original work is properly cited. 
and hemiparesis was found on the left side of grade IV.

Brain computed tomography scan showed no hemorrhagic findings, and brain magnetic resonance imaging scan showed acute ischemic stroke with high-signal intensity in diffuse-weighted images and low-signal intensity in apparent diffusion coefficient in the right pons (Fig. 1).

On the magnetic resonance angiography image, both middle cerebral arteries were occluded (Fig. 2), and moyamoya disease with bilateral distal ICA occlusion and collateral circulation from the neovascular structure and external carotid artery branch was confirmed through conventional angiography (Fig. 3).

The patient was treated with dual antiplatelet therapy with aspirin and clopidogrel and is undergoing physical rehabilitation therapy.

\section{DISCUSSION}

Ischemic stroke rarely occurs after a bee sting or drug-induced
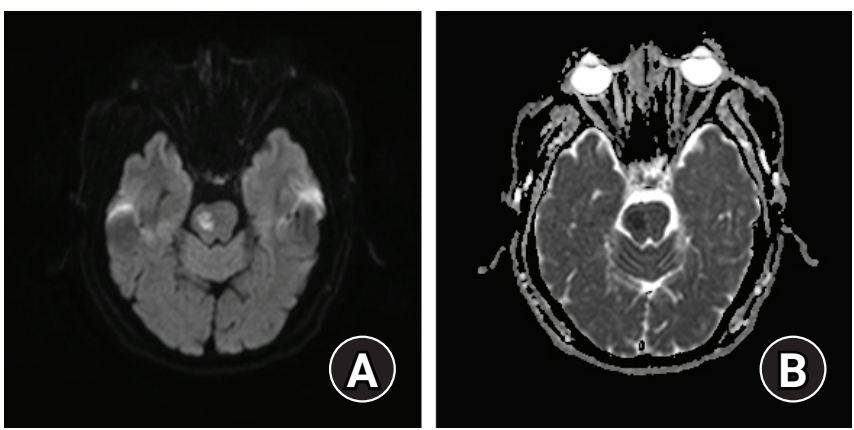

Fig. 1. (A) Diffusion-weighted magnetic resonance image shows high signal intensity on the right pons. (B) Apparent diffusion coefficient shows low signal in the same area. anaphylaxis, but a few such cases have been reported ${ }^{5,6)}$. However, the cause of ischemic stroke can be easily predicted based on a simple allergic reaction without anaphylactic shock due to a poor cerebrovascular supply caused by moyamoya disease. Despite posterior circulation involvement in moyamoya disease has not been well studied, it is likely an extension of the stenotic process from the anterior circulation ${ }^{7)}$. The allergic reaction may be caused by transient broad peripheral vasodilatation and collapsed fragile cerebrovascular network.

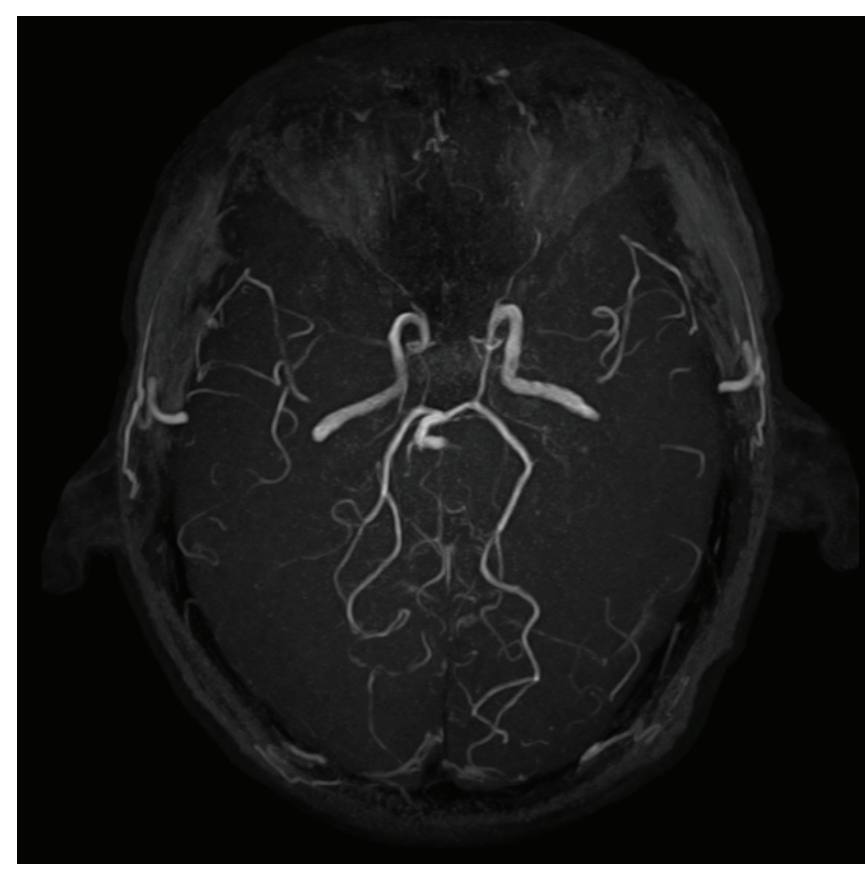

Fig. 2. In the magnetic resonance angiography, both distal ICAs were occluded. Also both anterior cerebral arteries and middle cerebral arteries were disappeared.
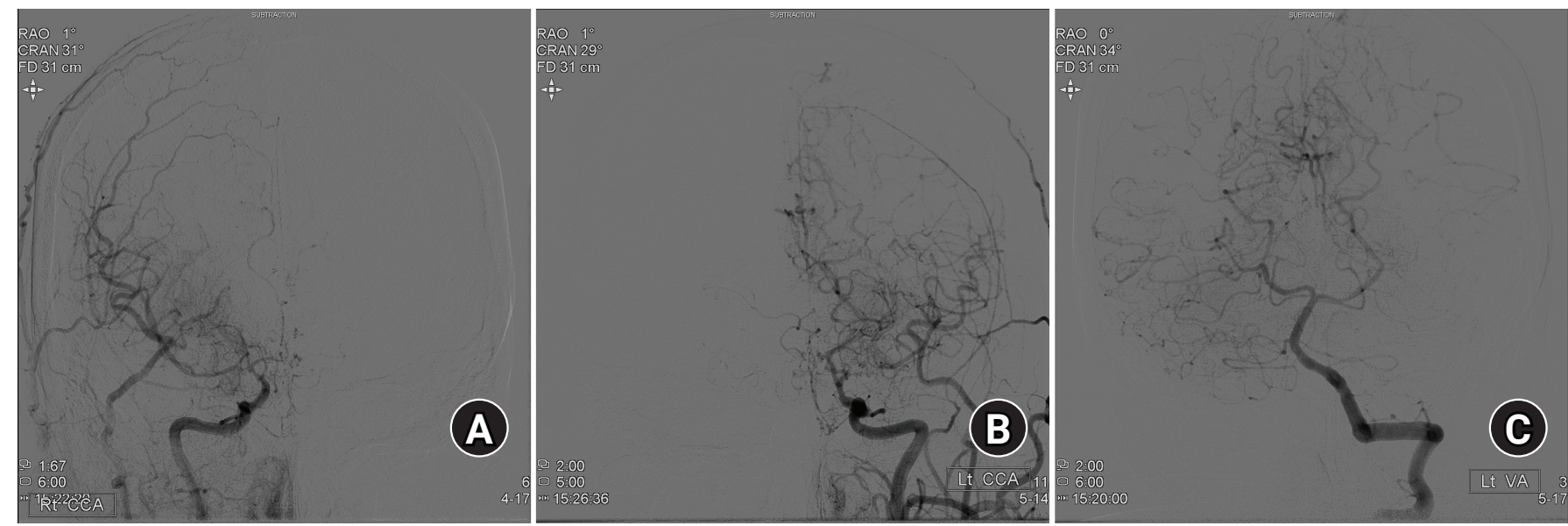

Fig. 3. (A, B) In the cerebral angiography, the moyamoya blood vessels in the form of "puff of smoke" are visible in the both middle cerebral artery territories. (C) Both posterior cerebral arteries form collateral circulation to the both cerebral hemisphere. 
Additionally, an allergic reaction possibly causes autonomic dysfunction8), which result in transient hypotension and ischemic stroke and can also be a means to rationally explain the developmental process ${ }^{9}$.

The prognosis of moyamoya disease can lead to contradictory results of hemorrhagic or ischemic stroke; however, no methods have been able to predict it yet ${ }^{3)}$.

\section{CONCLUSIONS}

This case report intended to provide evidence that an allergic reaction without anaphylaxis can cause ischemic stroke in patients with moyamoya disease, which is considered to be a significant point for both patients and neurologists.

\section{NOTES}

\section{Conflict of interest}

No potential conflict of interest relevant to this article was reported.

\section{REFERENCES}

1. Fukui M. Guidelines for the diagnosis and treatment of spontaneous occlusion of the circle of Willis ("moyamoya" disease). Research committee on spontaneous occlusion of the circle of willis (moyamoya disease) of the ministry of health and welfare, Japan. Clin Neurol Neurosurg 1997;99(Suppl 2):105-118.

2. Bersano A, Guey S, Bedini G, Nava S, Hervé D, Vajkoczy P, et al. Research progresses in understanding the pathophysiology of moyamoya disease. Cerebrovasc Dis 2016;41:105-118.

3. Canavero I, Vetrano IG, Zedde M, Pascarella R, Gatti L, Acerbi F, et al. Clinical management of moyamoya patients. J Clin Med 2021;10:3628.

4. Reber LL, Hernandez JD, Galli SJ. The pathophysiology of anaphylaxis. J Allergy Clin Immunol 2017;140:335-348.

5. Robles LA, Matilla AF. Brain stem ischemic stroke associated with anaphylaxis. Cureus 2018;10:e2289.

6. Brown AF, McKinnon D, Chu K. Emergency department anaphylaxis: a review of 142 patients in a single year. J Allergy Clin Immunol 2001;108:861-866.

7. Fox BM, Lawton MT, Wanebo JE. Pathophysiology of vascular stenosis and remodeling in moyamoya disease. Front Neurol 2021; 12:661578.

8. Loehrl TA. Autonomic dysfunction, allergy and the upper airway. Curr Opin Otolaryngol Head Neck Surg 2007; 15:264267.

9. Machida T, Higuchi Y, Nakano S, Ishige S, Shimada J, Honma K. Postoperative cerebral ischemia due to hypotension in moyamoya patient with autonomic dysfunction. World Neurosurg 2018;109:204-208. 